\title{
Motor intention decoding during active and robot-assisted reaching
}

\author{
Aldo Pastore*, Camilla Pierella*, Fiorenzo Artoni, Elvira Pirondini, Martina Coscia, Maura Casadio, \\ and Silvestro Micera, Member, IEEE
}

\begin{abstract}
Robotics rehabilitation is a widely used approach for the treatment of patients with severe motor disabilities, such as stroke survivors. Robots can provide intense, controlled and repeatable rehabilitation and they can also provide different levels of assistance when patients are not able to initiate or complete a movement. Nevertheless, several studies proved that completely passive movements are not sufficient to stimulate neuro-motor recovery and patients' engagement is a key factor for an effective rehabilitation. For this reason it is important to combine techniques for detection of movement intention (MI) with rehabilitation robotics. In this study we developed an algorithm capable of detecting MI before the movement onset, in order to obtain a trigger signal for providing robotics assistance. The proposed algorithm automatically selects the channels used to extract MI based on the motor-information content of each channel. The developed algorithm was tested on data recorded on $n=8$ healthy subjects performing 3D reaching movements with an exoskeleton in active and assisted conditions. MI was detected about $400 \mathrm{~ms}$ before the beginning of the movement and the performance of the proposed method were significantly higher than the one achieved when six preselected channels, located over motor areas, were used for MI decoding. MI was also detected during robot-assisted movements. Interestingly, in active movements the highest performance was achieved with electrodes over a well-localized cluster above the contralateral and central motor areas, while in passive executions, the areas with the best performances became more sparse.
\end{abstract}

\section{INTRODUCTION}

Rehabilitative robotics is widely used for motor recovery after neurological accidents, such as stroke. Indeed, robotic systems are capable to deliver assistance and therapy in an extended, adaptable and repeatable way. Several studies [1, 2] highlighted the importance of an intensive motor training, with a high number of movement repetitions, to increase the rehabilitative effectiveness of therapy, therefore robots seem

* equal contribution

Research supported by the European Union's Horizon 2020 research and innovation program under the H2020 Marie Skłodowska-Curie Actions Grant Agreement No.750947-BIREHAB

A. Pastore and M. Casadio are with the University of Genova, 16145 Genova, Italy (maura.casadio@unige.it)

C. Pierella, F. Artoni and S. Micera are with the Bertarelli Foundation Chair in Translational Neuroengineering, Center for Neuroprosthetics and Institute of Bioengineering, École Polytechnique Fédérale de Lausanne (EPFL), 1202 Geneva, Switzerland (camilla.pierella@epfl.ch, fiorenzo.artoni@epfl.ch, silvestro.micera@epfl.ch)

E. Pirondini is with the Department of Radiology and Medical Informatics, University of Geneva, and with the Institute of Bioengineering/Center for Neuroprosthetics, Ecole Polytechnique Fédérale de Lausanne (EPFL), 1202 Geneva, Switzerland (Elvira.Pirondini@unige.ch)

M. Coscia is with the Wyss Center for Bio- and Neuroengineering, 1202 Geneva, Switzerland (Martina.Coscia@wysscenter.ch) to fit well the goal of helping restoring compromised motor functions. The first systems adopted in upper limb rehabilitation were robotic manipulators that guided the hands of patients during 2-D exercises specifically designed to stimulate the recovery of the injured limb [3-5]. Subsequently, new and more sophisticated types of devices were developed: exoskeletons are among these. In particular exoskeletons [6] are able to guide the movement of the entire limb, controlling degrees of freedom that standard 2-D end-effector robots cannot actuate. With rehabilitative robots the therapy can be executed in different modalities: active, the patient is actively moving; passive, the robot provides assistance to move and the patient's active efforts are not considered; assistance-as-needed, robot provides assistance as needed based on the patient's performance.

It has been proved that during passive rehabilitation tasks active engagement and sensorimotor stimulation are lacking, resulting in a less effective motor recovery outcome $[7,8]$. Indeed, although the robotic assistance has the advantage to make possible a long, repeatable and intense therapy, if patients are not actively involved in the task the effect of robotics rehabilitation is not sufficient to improve the recovery process $[7,8]$. The assistance-as-needed modality seems to be a better way to perform a rehabilitation session with a robot. Following the Hebbian plasticity principle [9], which states that the shorter the delay between input and response the bigger the process of plasticity, the timing of the assistance delivery is one of the most important aspects. A time delay between movement intention (MI) and assistance delivery that is not short enough is not capable to stimulate synaptic plasticity for functional recovery. Current methods to engage patients more actively in their therapy include triggering assistance based on force, velocity, or time thresholds, or detecting MI based on electromyography (EMG) or electroencephalography (EEG)[10]. Nevertheless, the first three methodologies are not appropriate for patients that are not able to exert forces. In these cases, a common approach is to deliver assistance after a fixed period of time, assuming the patient is attempting to move even if he/she is not moving at all and, thus, violating the Hebbian principle [5]. Alternatively, when the movement is visually absent, motor intention can be detected through neural signals, such as EMG or EEG. The first is a direct consequence of the latter, hence to minimize the delay between assistance delivery and MI, the EEG seems to be the optimal choice to detect MI.

The EEG signal is the activity produced by cortical neurons and directly recorded by superficial electrodes placed on the scalp. It carries information about motor processes, therefore several authors had tried to use it for non-invasive brain machine interfaces (BMIs) $[9,11,12]$ proving the efficacy of using EEG and BMIs for rehabilitation $[13,14]$. What makes a BMI interesting, in 
this field, is the possibility to detect signals produced by the brain strictly related to the attempt of moving, that is the clear evidence of subjects' engagement in rehabilitative tasks. Moreover, there is an additional requirement for enhancing motor recovery: the timing of the robotic assistance delivery $[14,15]$. Indeed, in a recent work [16] it has been demonstrated that to promote neural plasticity as consequence of external stimulations to facilitate movement generation, it is fundamental that the stimulation is provided simultaneously to the imagination or the attempt to move. Considering all these aspects and criticalities about rehabilitation robotics, BMIs and their possible combinations to enhance plasticity and motor recovery, in our work we designed and developed an algorithm capable to detect motor intention with low delay based on readiness potentials (RP), from the most informative channels. RP is an unconscious preparatory activity, belonging to the family of slow cortical potentials (SCPs). Its main component is characterized by a steep negative slope focused over the contralateral motor area, that begins about $400 \mathrm{~ms}$ before movement onset [17]. It is considered a manifestation cortical contribution to the pre-motor planning of volitional movements and it has been already extensively used in the BMI field [12, 18, 19] to decode movement intention. We tested our algorithm to detect MI on data recorded from subjects that active reaching movements while wearing an exoskeleton. Then we verified if and to what extent these neural processes related to voluntary movements were still present in the robot-assisted movements.

\section{MATERIALS AND METODS}

\section{A. Experimental set-up}

Data used in this study were recorded during a roboticassisted experiment involving 8 healthy male subjects (age: $24.42 \pm 1.81$ ) [20]. The participants were going through a training session using the Arm Light Exoskeleton (ALEx, Wearable Robotics) [21]. ALEx is a mechanically compliant powered exoskeleton for the right upper limb; it allows movements in a 3D workspace both in active and in passive modalities. In the former subjects moved to the target autonomously wearing the exoskeleton; gravity, inertia and friction were compensated. Instead, in the passive modality an additional force was provided to the subject, whose right hand was passively moved in order to reach targets. The robotic assistance provided in the passive modality followed a minimum jerk profile, as reported in [22].

During the experiment, subjects wore ALEx and seated in front of a panel, displaying eight targets arranged in a clock fashion; the center of the panel was aligned to the subject's acromion. Every trial started with a sound cue provided to the subject indicating the target to reach. Then the subject executed the reaching movements from the center to one of the eight targets, and then they returned to the starting position. The temporal distance between the sound cue and the movement start was $1.23 \pm 0.11 \mathrm{~s}$ for active reaching and $1.8301 \pm 0.06 \mathrm{~s}$ for the robot assisted reaching. The training consisted in ten blocks of eight trials (center-out reaching movements), each one to a different target selected in a random order. The ten blocks of movements were executed first in active and then in the passive modality.

\section{B. Data recording and pre-processing}

Position and speed of the end-effector and of the articular joints were recorded by ALEx at $100 \mathrm{~Hz}$. For the entire duration of the experiment EEG data were also acquired using a 64 channels Active-Two system with standard 10-20 configuration (Biosemi, Amsterdam Netherlands) at a sampling rate of $2048 \mathrm{~Hz}$. The exoskeleton and the EEG systems were synchronized using a trigger signal sent from ALEx every time one of the following event occurred: acoustic cue, movement start, movement end. The start and the end points of each reach were defined as the time points when the speed profile of the $\mathrm{EE}$ of the exoskeleton exceeded or dropped below $2 \%$ of the local maximum value[21].

Pre-processing of the raw EEG signal was conducted using Brainstorm [23]. The EEG was first filtered using a band-pass filter with cut-off frequencies between 0.1 and 30 $\mathrm{Hz}$ (FIR filter, order 572, Kaiser-Bessel window: Brainstorm band-pass relaxed filter) and down sampled from $2048 \mathrm{~Hz}$ to $128 \mathrm{~Hz}$ (Brainstorm resample function). The next step consisted in a manual examination of each channel of the whole session for each subject in order to exclude noisy epochs. Moreover, only 38 out 64 channels were maintained, excluding the most external ones (see Figure 5 for reference to the channels considered). This operation was done to remove the signals recorded by electrodes that lied over regions far from motor areas, and that carried a lot of muscular noise. Subsequently, Infomax ICA [24] was applied to eliminate components relate to blinks and eye movements as in [25]. Finally, to decode motor intention with the purpose of minimizing the detection delay we extracted the readiness potential (RP) or Bereitschafts potential [26] To better capture this slow signal is important to filter the EEG between 0.1 and $1 \mathrm{~Hz}$ (FIR filter, order 572, Kaiser-Bessel window: Brainstorm band-pass relaxed filter). [19]. Thus the recordings were band-pass filtered between that frequency interval and epoched. The epoching procedure was performed using the events generated by ALEx, in particular the sound cue trigger and the movement start trigger. We extracted a 'baseline interval', i.e. a period that ranged between $500 \mathrm{~ms}$ before the sound and the sound itself, where the EEG records were not supposed to be influenced by the motor task. Then a larger segment was extracted from every trial that goes from $2000 \mathrm{~ms}$ to 1500 ms after the start of the movement.

\section{Decoding Motor intention}

For the motor intention decoding, a two-classes Linear Discriminant Analysis (LDA) algorithm was adopted. The class 1 was defined as the 'motor intention class' and class 0 as the 'no motor intention class'. For every subject and condition, training and test sets were randomly defined selecting the $75 \%$ and $25 \%$ of that whole specific group of trials, respectively. We then defined the examples to use for training the MI classifiers; in particular, from each trial of the training set three windows of $500 \mathrm{~ms}$ used as examples 


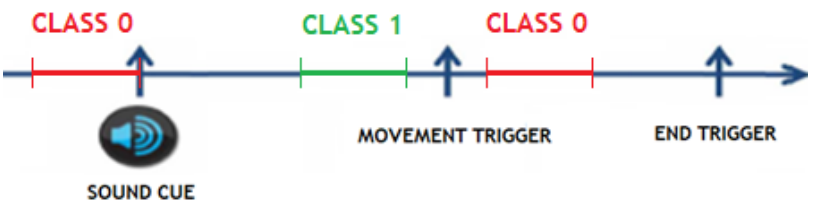

Figure 1. Cartoon showing the three events (vertical arrows) happening during a trial, sound cue, movement start, movement end, and the three regions of interest. Along the timeline, the $500 \mathrm{~ms}$ windows assigned to the class 0 or 'no movement intention' are highlighted in red; the one assigned to the class 1 or 'movement intention' is instead highlighted in green.

of the two classes. The features of each example were the eight, linearly spaced, temporal points within the corresponding window. For each trial, the window from 750 $\mathrm{ms}$ to $250 \mathrm{~ms}$ before the movement onset was associated to the 'motor intention' class [12]. The remaining two windows were assigned to the 'no motor intention' class: one was composed by the $500 \mathrm{~ms}$ of baseline interval, and the other ranged between $250 \mathrm{~ms}$ and $750 \mathrm{~ms}$ after the movement start. In Figure 1 it is showed a typical trial and the three windows selected as example.

The algorithm that we developed is based on the automatic selection of the channels to be used for the classification; the channels were singularly selected for each subject and condition in order to be subject-specific. First, a subset of channels carrying significant information was identified; with this objective a multivariate ANOVA was performed using every example from the training set, evaluating the capacity of each channel to discriminate between the 'motor intention' and the 'no motor intention' class. This algorithm was executed on the whole set of 38 channels; therefore each one of them obtained a p-value. Subsequently, a p-value threshold was fixed and the channels that obtained a p-value higher than the threshold were excluded from the following steps. The threshold consisted in the 'knee point' calculated on the logarithm of the p-values achieved by each channel, sorted in ascending order.

For each subject, the channels that obtained a p-value lower or equal than the knee point were used singularly to train an LDA classifier, using only examples of the training set. To test the trained classifiers we used a shifting window method, in order to simulate the real-time application of the decoding algorithm. Specifically, for each trial of the test set the steps were the following: selecting the first $500 \mathrm{~ms}$ from the corresponding channel, down sampling it to $16 \mathrm{~Hz}$ to form an example, pass the example to the classifier to obtain a binary classification value ( 0 or 1 , respectively if example was classified as no motor intention or motor intention) and finally assigning this classification value to the last temporal point in the window [12] (for example $-1500 \mathrm{~ms}$ if the window ranged between $-2000 \mathrm{~ms}$ and $-1500 \mathrm{~ms}$ ). After these operations the window was shifted forward of $40 \mathrm{~ms}$ and each step was performed again with the new window until the end of the trial. For each trial, a binary function was therefore obtained. At the end, we averaged the single trial results obtaining a mean classification result for each subject and condition. As in [12] the detection was considered to start when the average classification function was above the chance level, for five consecutive temporal points. In this work the chance level is considered to be $50 \%$ due to the binary nature of the problem.

To assess which channel, among those selected in point 2 , obtained the best decoding performance we designed a 'score function'. This function was composed of three parts, one with positive and two with negative values. Specifically it was positive in the temporal range between $500 \mathrm{~ms}$ before the movement onset and the onset itself, i.e. the interval in which the MI should be identified. The two negative sides instead ranged between $1500 \mathrm{~ms}$ and $540 \mathrm{~ms}$ before movement, and between $40 \mathrm{~ms}$ and $1500 \mathrm{~ms}$ after movement. A negative score was therefore assigned to these regions where the MI was not supposed to be present. The choice to use the $4^{\text {th }}$ polynomial for the negative segments was made considering the fact that the precise temporal localization of movement intention is not known, i.e. only an interval can be defined. Thus, since the points closest to the negative-positive borders might be near to motor intention temporal localization their negative contributes to the score must be lower. Additionally, the left side of the negative region was multiplied by 0.75 while the right one by 0.25 , before the computation of the score; the different weights distribution was decided considering that in a hypothetical on-line scenario, errors in movement intention decoding located before the movement start would be the worst because they would compromise the timing of the roboticassistance. The positive segment of the score function was moreover normalized by the sum of all its values, instead the two negative segments were normalized by the sum of values inside both of them. This function is analytically described in (1) and showed in Figure 2:

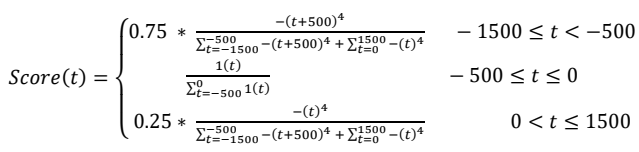

where $t$ is the time.

For each channel we then computed the score, as the sum of the product between the normalized score function and the mean classification result of that channel. Thus the score ranged between -1 and 1 because of the normalization applied to the score function. The channel that achieved the highest score, for each subject and movement modality, was

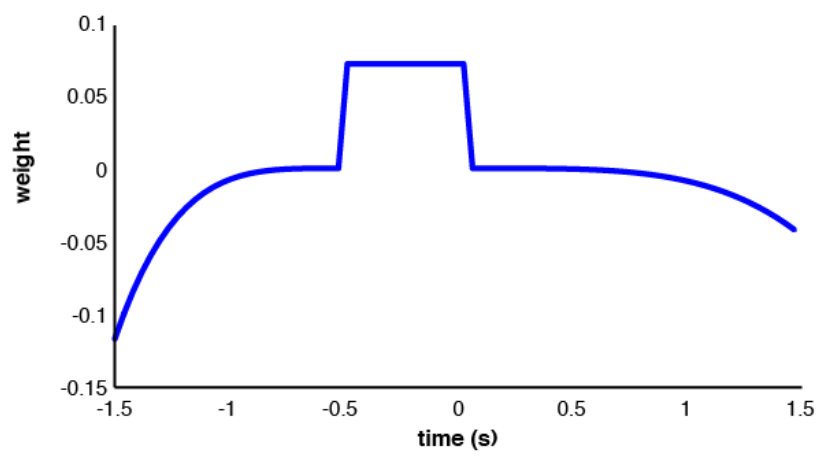

Figure 2. The normalized score function defined in order to evaluate the results of the classification. 
labeled as 'the best channel'. This one was used in our 'best channel classifier'.

After assigning a score to each channel, a second LDA classifier was trained using the channels that obtained a score value at least equal to half of the maximum, we called it 'average channel classifier'. Each trial of the test set was averaged over the selected channels in order to obtain an 'average channel'. Moreover, each channel used in this operation contributed in different way in accordance with its score. The computation of the average channel is described in equation (2). The trained classifier was subsequently tested using the shifting window method. A score value was computed even for the average channel, in the same way as described for the physical ones.

$$
\text { Average Channel }(t)=\frac{\sum_{\text {channel }=1}^{\text {Nchannels }} \text { Trial }(t, \text { channel }) * \text { Score }(\text { channel })}{\text { Nchannels }}
$$

with Nchannels equal to the number of channels that obtained a score value at least equal to half of the maximum and $t$ is the time. After the classification, each EEG channel was identifiable by its score. We then performed a cluster analysis in order to verify whether the more informative channels (i.e. the ones with higher score) were clustered on meaningful areas, i.e. motor and premotor areas. We used a k-means algorithm in order to identify two clusters, in particular the cluster with the channels that obtained a high score and a cluster with the ones that resulted having low scores. Therefore each electrode was clustered using its 2D spatial position, and its score index in a 256-colormap, i.e. the linear transformation of the score in a value that ranged between 0 and 256. This clustering procedure was performed for both training conditions, i.e., active and passive.

To compare the results of the new developed classification method based on an automatic selection of the channels, with a more standard procedure, we trained a third LDA classifier, the 'six channels classifier', using six preselected channels concatenated together. These channels were the same used in [12] and specifically they were $\mathrm{Cz}$, $\mathrm{C} 1, \mathrm{C} 2, \mathrm{Cpz}, \mathrm{CP} 1, \mathrm{CP} 2$, which are located over motor and somatosensory cortical regions. As for the two preceding methods, the shifting window method was used to test classification accuracy and subsequently the score was computed for the average classification result as well.

\section{E. Statistics}

All the statistical analysis was computed within Statsoft environment (Statistica software 7.1, Statsoft TULSA, USA). To assess the differences between the three classification methods we run a repeated measures analysis of variance (rANOVA), considering as within-subjects' factors: 'task condition' (1-2: Active, Passive) and 'classification method' (1-3: best channel, average channel, six fixed channels). The assumption of sphericity was tested on each variable using Mauchly's test. If the assumption was rejected the Greenhouse-Geisser correction was applied. Consequently a post-hoc analysis (Fisher's LSD test) was performed to better understand the differences emerged by the a priori analysis. First, each classification method in the active group was compared with its analogous of the passive group; after this, the classification types were compared between them across the same task condition.

To assess if the scores of the electrodes in the active best cluster, i.e. where motor intention was better detected, were different from the same one of the passive condition, a rANOVA with two within-subjects' factors: 'task condition' (1-2: Active, Passive) and 'channel' (1-7: F1, FC1, C1, CPz, $\mathrm{Fz}, \mathrm{FCz}, \mathrm{Cz}$ ) was run. The assumption of sphericity was tested and eventually the Greenhouse-Geisser correction was applied. Further, a post-hoc analysis (Fisher's LSD test) was used to investigate which of the seven channels mainly influenced the statistically significant differences obtained with the rANOVA. Finally, each channel used for the rANOVA test was individually compared between the active and the passive condition.

\section{RESULTS}

\section{A. Classification methods comparison}

We used the score obtained using each one of the three classification methods to assess which of them performed best in movement intention decoding, and if the task modality influenced the score. A significant main effect of the training conditions (active and passive, $\mathrm{F}(1,7)=7.7$, $\mathrm{p}=0.027)$ and of the classification methods $(\mathrm{F}(2,14)=23.8$, $\mathrm{p}<0.001)$ were found.

Within the same task condition, the best channel and average channel classification had similar performances both in active $(p=0.213)$ and in passive $(p=0.323)$ conditions. Moreover, each of them performed significantly better with respect to the fixed channels method (comparison between fixed channels method and (i) the best channel: active $\mathrm{p}<0.001$, passive $\mathrm{p}=0.0017$; (ii) average channel: active $\mathrm{p}<0.001$, passive $\mathrm{p}=0.013$ ). All the classification comparisons are summarized in Figure 3.

When comparing the same classification method across different task conditions, it emerged that the 'best channel' and the 'average channel' methods performed significantly better when the subject was actively moving $(\mathrm{p}<0.001)$, but no difference was found when the fixed channels method was used $(\mathrm{p}=0.179)$.

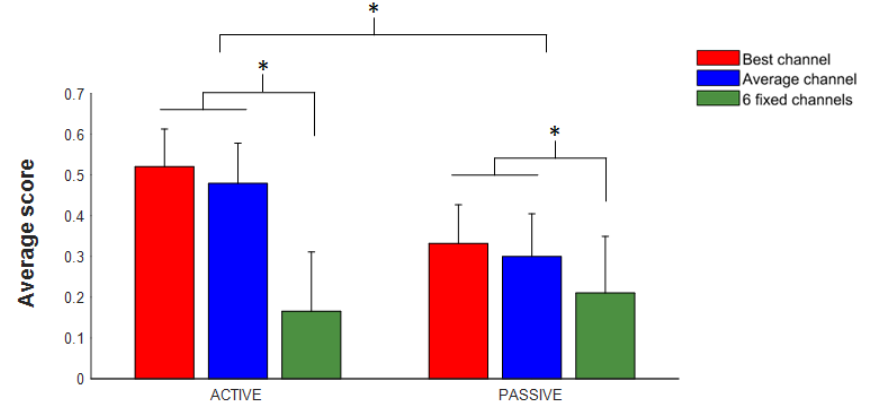

Figure 3. Mean and standard deviation of score values across the whole population for each classification methods, in both active (left) and passive (right) task executions. The red, blue, and green bars represent respectively the 'best channel' modality, the 'average channel' modality and the ' 6 fixed channels' modality. 


\section{B. Classification curves using the 'best channel' classification method}

Since the 'best channel' classification method showed the overall best performance, additional details are reported below, showing the classification rates curves and the correspondent detection time. Figure $4 \mathrm{a}$ and $4 \mathrm{~b}$ show the classification rates for one of the eight subjects in both active and passive conditions using the best channel method, $\mathrm{C} 1$ for the subject active and CP3 for the subject passive. From the classification rate curve the score were calculated; the average scores achieved in active and passive condition were respectively $0.52 \pm 0.092$ and $0.33 \pm 0.095$. The motor intention continued to be considered as detected until the classification value remained above this level. The mean detection times were $-0.4014 \pm 0.0706 \mathrm{~s}$ in the active condition and $-0.3623 \pm 0.1225 \mathrm{~s}$ in the passive condition; this difference was not significantly different.

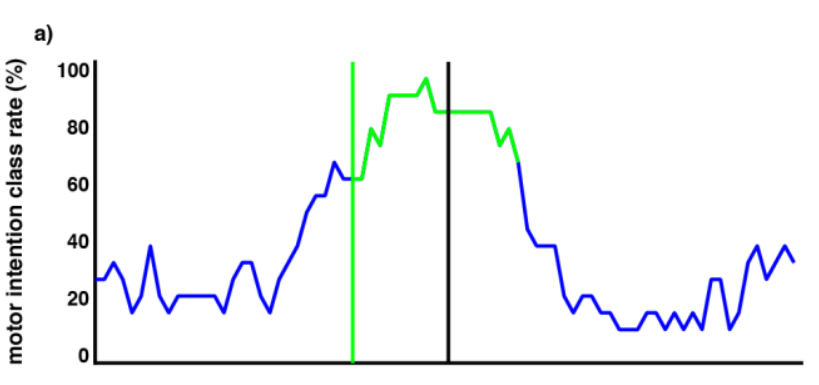

b)

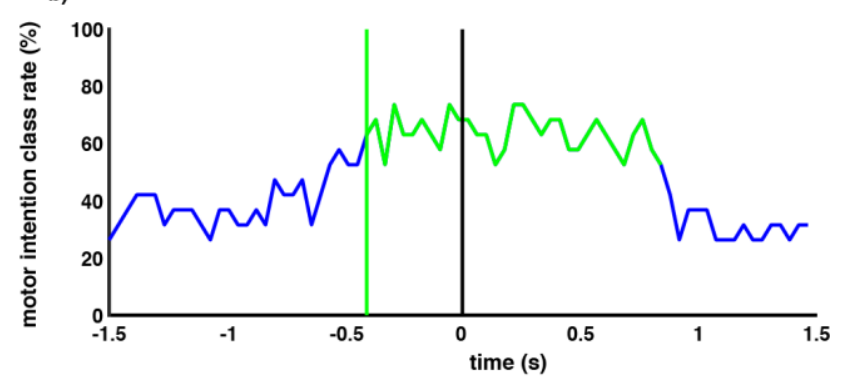

Figure 4. Classification rate curves for subject 8 during active (a) and passive (b) conditions. The vertical black lines indicate the movement onset and the vertical green lines show when the algorithm detected the beginning of movement intention. The classification curve colored in green indicates the interval during which the movement intention was detected.

\section{Topographic maps of the score spatial distribution}

Additional analyses on the classification were done in order to understand the spatial location of the channels selected as 'best channel'. Results showed that 7 out of 8 subjects, in active condition, had high classification scores over the fronto-central and central channels (primary and supplementary motor cortex) of the midline and contralateral side; 6 out 8 had also the best channel in this area. In the passive condition instead the best channel of each subject did not have a recurrent spatial localization, but in general it was slightly shifted towards parietal regions. Figure 5 shows the average topographic maps of scores for active and passive conditions.

From the cluster analysis, showed in figure 6 , it resulted that the best cluster during active movements was mainly located over the central and contralateral motor cortex (primary and supplementary), in particular composed of

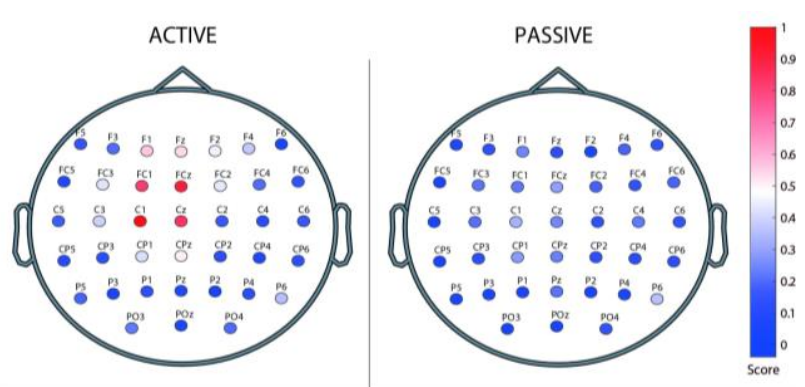

electrodes $\mathrm{F} 1, \mathrm{FC} 1, \mathrm{C} 1, \mathrm{CPz}, \mathrm{Fz}, \mathrm{FCz}, \mathrm{Cz}$; the best cluster during passive movements was instead formed by electrodes F1, FC3, FC1, C1, C3, CP1, Pz, CPz, F4, FC6, FC2, FCz, $\mathrm{Cz}, \mathrm{C} 4, \mathrm{P} 6$. Indeed it was less spatially concentrated, even if a consistent part of it was still located over contralateral motor areas.

Figure 5. Topographical maps of the average score assigned to each channel in active (left) and passive (right) task conditions for the whole population. The average score value of every channel is normalized by the maximum average score of the grand average active map.

Finally, we tested if the scores of the electrodes in the cluster where motor intention was better detected during the active movement tasks were different in passive and active condition. We found a significant difference $(F(1,7)=10.8$, $\mathrm{p}=0.013$ ) and proceeded with post-hoc analysis (the 'channel' factor was at the threshold of significance $(\mathrm{F}(6,49)=3.29, \mathrm{p}=0.05)$. Comparing each channel located in this cluster between the active condition and the passive one, we found that every channel carried significant differences, at least $\mathrm{p}<0.05$. Additionally, there were four of them that achieved a $\mathrm{p}<0.001$, i.e. FC1, C1, Cz, FCz.
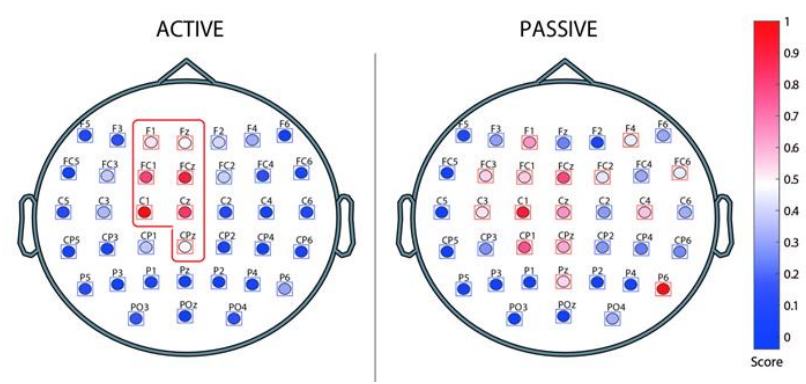

Figure 6. Topographical maps of the average score associated to each channel in active (left) and passive (right) task conditions for the whole population of subjects. Both in the active and passive map, the score value of every channel is normalized by the average score of the channel that achieved the maximum value in the corresponding task condition. In both maps, red squares are drown around the channels resulted the best channels, from cluster analysis, and blue squares around the ones resulted the worst. Moreover the best cluster in active condition is highlighted by the red line.

\section{CONCLUSION}

The main goal of this study was to design and develop an EEG-based algorithm for brain machine interface that could detect the motor intention before the movement onset, with minimum delay, in order to have a reliable trigger to provide assistance during robot-based rehabilitation therapy. We developed a subject-specific method that automatically selected the channels that better detected the motor intention phenomenon and we compared it with a method based on six preselected channels. Moreover, to investigate if a voluntary intention to move was present and detectable even when a 
subject executed a robot-assisted reaching, we tested the algorithm using data from subjects that performed both active and passive movements wearing ALEx. From the results we found a significant difference between decoding performance in active and passive conditions. Specifically in the passive one, the classification results were lower, meaning that the subject's involvement in the task was low. However, the classification results were still above chance level. Subsequently, we examined the spatial distributions of the score across subjects in the two reaching modalities. In the active modality the channels with the highest classification values were organized in a clear cluster mainly located over the contralateral and central motor cortex. The cluster of best channels in the passive condition became less localized and shifted towards parietal areas, even if the main part was still on the contralateral motor cortex. Hence, the precise clustering of scores over motor areas in active movements reflects the fact that neural correlates related with the movement intention was detected; on the other hand results for the passive condition underlines the lack of involvement in the motor task that could make the robotassisted motor rehabilitation ineffective.

Indeed, this algorithm, in an on-line version, could be used to monitor subject's involvement during robotic rehabilitation tasks, in order to provide assistance only if a voluntary moving attempt is detected. Nevertheless the classification algorithm that we developed, could detect motor intention also during passive movement, even if with lower performance, showing its potential use also with people that suffer of sever paralysis. Finally, future works could combine features extracted from muscular activity to improve the motor intention detection [27].

\section{REFERENCES}

[1] G. Kwakkel, R. C. Wagenaar, T. W. Koelman, G. J. Lankhorst, and J. C. Koetsier, "Effects of intensity of rehabilitation after stroke. A research synthesis," Stroke, vol. 28, pp. 1550-6, Aug 1997.

[2] K. Wing, J. V. Lynskey, and P. R. Bosch, "Whole-body intensive rehabilitation is feasible and effective in chronic stroke survivors: a retrospective data analysis," Top Stroke Rehabil, vol. 15, pp. 247-55, May-Jun 2008.

[3] H. I. Krebs, N. Hogan, M. L. Aisen, and B. T. Volpe, "Robot-aided neurorehabilitation," IEEE Trans Rehabil Eng, vol. 6, pp. 75-87, Mar 1998.

[4] P. S. Lum, D. J. Reinkensmeyer, and S. L. Lehman, "Robotic assist devices for bimanual physical therapy: preliminary experiments," IEEE Transactions on Rehabilitation Engineering, vol. 1, pp. 185191, 1993.

[5] R. Colombo, F. Pisano, S. Micera, A. Mazzone, C. Delconte, M. C. Carrozza, et al., "Robotic techniques for upper limb evaluation and rehabilitation of stroke patients," Ieee Transactions on Neural Systems and Rehabilitation Engineering, vol. 13, pp. 311-324, Sep 2005.

[6] H. S. Lo and S. Q. Xie, "Exoskeleton robots for upper-limb rehabilitation: state of the art and future prospects," Med Eng Phys, vol. 34, pp. 261-8, Apr 2012.

[7] N. Hogan, H. I. Krebs, B. Rohrer, J. J. Palazzolo, L. Dipietro, S. E. Fasoli, et al., "Motions or muscles? Some behavioral factors underlying robotic assistance of motor recovery," J Rehabil Res Dev, vol. 43, pp. 605-18, Aug-Sep 2006.

[8] H. I. Krebs, B. Volpe, and N. Hogan, "A working model of stroke recovery from rehabilitation robotics practitioners," J Neuroeng Rehabil, vol. 6, p. 6, Feb 252009.

[9] N. Jiang, N. Mrachacz-Kersting, R. Xu, K. Dremstrup, and D. Farina, "An accurate, versatile, and robust brain switch for neurorehabilitation," in Brain-Computer Interface Research, ed: Springer, 2014, pp. 47-61.

[10] A. A. Blank, J. A. French, A. U. Pehlivan, and M. K. O’Malley, "Current Trends in Robot-Assisted Upper-Limb Stroke Rehabilitation: Promoting Patient Engagement in Therapy," Current Physical Medicine and Rehabilitation Reports, vol. 2, pp. 184-195, September 012014.

[11] T. P. Luu, S. Nakagome, Y. He, and J. L. Contreras-Vidal, "Real-time EEG-based brain-computer interface to a virtual avatar enhances cortical involvement in human treadmill walking," Sci Rep, vol. 7, p. 8895, Aug 212017.

[12] E. Lew, R. Chavarriaga, S. Silvoni, and R. Millan Jdel, "Detection of self-paced reaching movement intention from EEG signals," Front Neuroeng, vol. 5, p. 13, 2012.

[13] M. A. Nicolelis, "Brain-machine interfaces to restore motor function and probe neural circuits," Nat Rev Neurosci, vol. 4, pp. 417-22, May 2003.

[14] A. Ramos-Murguialday, D. Broetz, M. Rea, L. Laer, O. Yilmaz, F. L. Brasil, et al., "Brain-machine interface in chronic stroke rehabilitation: a controlled study," Ann Neurol, vol. 74, pp. 100-8, Jul 2013.

[15] M. Takahashi, K. Takeda, Y. Otaka, R. Osu, T. Hanakawa, M. Gouko, et al., "Event related desynchronization-modulated functional electrical stimulation system for stroke rehabilitation: a feasibility study," J Neuroeng Rehabil, vol. 9, p. 56, Aug 162012.

[16] N. Mrachacz-Kersting, S. R. Kristensen, I. K. Niazi, and D. Farina, "Precise temporal association between cortical potentials evoked by motor imagination and afference induces cortical plasticity," $J$ Physiol, vol. 590, pp. 1669-82, Apr 12012.

[17] H. Shibasaki and M. Hallett, "What is the Bereitschaftspotential?," Clin Neurophysiol, vol. 117, pp. 2341-56, Nov 2006.

[18] N. Birbaumer, N. Ghanayim, T. Hinterberger, I. Iversen, B. Kotchoubey, A. Kubler, et al., "A spelling device for the paralysed," Nature, vol. 398, pp. 297-8, Mar 251999.

[19] G. Garipelli, R. Chavarriaga, and J. D. Millan, "Single Trial Recognition of Anticipatory Slow Cortical Potentials: The Role of Spatio-Spectral Filtering," 2011 5th International Ieee/Embs Conference on Neural Engineering (Ner), pp. 408-411, 2011.

[20] A. Sadaka-Stephan, E. Pirondini, M. Coscia, and S. Micera, "Influence of trajectory and speed profile on muscle organization during robot-aided training," Proceedings of the Ieee/Ras-Embs International Conference on Rehabilitation Robotics (Icorr 2015), pp. 241-246, 2015.

[21] E. Pirondini, M. Coscia, S. Marcheschi, G. Roas, F. Salsedo, A. Frisoli, et al., "Evaluation of the effects of the Arm Light Exoskeleton on movement execution and muscle activities: a pilot study on healthy subjects," J Neuroeng Rehabil, vol. 13, p. 9, Jan 232016.

[22] R. Iandolo, V. Squeri, D. De Santis, P. Giannoni, P. Morasso, and M. Casadio, "Testing proprioception in intrinsic and extrinsic coordinate systems: is there a difference?," 2014 36th Annual International Conference of the Ieee Engineering in Medicine and Biology Society (Embc), pp. 6961-6964, 2014.

[23] F. Tadel, S. Baillet, J. C. Mosher, D. Pantazis, and R. M. Leahy, "Brainstorm: A User-Friendly Application for MEG/EEG Analysis," Computational Intelligence and Neuroscience, 2011.

[24] A. J. Bell and T. J. Sejnowski, "An information-maximization approach to blind separation and blind deconvolution," Neural Comput, vol. 7, pp. 1129-1159, 1995.

[25] F. Artoni, C. Fanciullacci, F. Bertolucci, A. Panarese, S. Makeig, S. Micera, et al., "Unidirectional brain to muscle connectivity reveals motor cortex control of leg muscles during stereotyped walking," Neuroimage, vol. 159, pp. 403-416, Aug 042017.

[26] H. H. Kornhuber and L. Deecke, "Brain potential changes in voluntary and passive movements in humans: readiness potential and reafferent potentials," Pflugers Arch, vol. 468, pp. 1115-24, Jul 2016.

[27] F. Artoni, E. Pirondini, A. Panarese, and S. Micera, "Exploring neuromuscular synergies of reaching movements with unified independent component analysis," Conf Proc IEEE Eng Med Biol Soc, vol. 2016, pp. 3183-3186, Aug 2016. 